\title{
The Amygdala Central Nucleus: A New Region Implicated in Habit Learning
}

\author{
Jean-Marie N. Maddux, Felipe L. Schiffino, and Stephen E. Chang \\ Department of Psychological and Brain Sciences, The Johns Hopkins University, Baltimore, Maryland 21218 \\ Review of Lingawi and Balleine
}

The mechanisms underlying instrumental conditioning have been a rich source of experimental inquiry. Perhaps one of the most influential findings has been the transition from goal-directed, outcome-guided responding early in training to habitual, stimulus-response behavior following extended training of the instrumental response (Adams, 1982). To assess which strategy is being used, outcome devaluation procedures have proven useful. In these procedures, the outcome is devalued either through selective satiation or pairing with lithium chloride ( $\mathrm{LiCl}$ ) injections that subsequently induce gastric malaise; instrumental responding on the manipulandum that had been trained to yield the outcome is then assessed, typically in extinction. Instrumental responding that reflects sensitivity to devaluation indicates goal-directed action, whereas instrumental responding that is insensitive to devaluation demonstrates habitual behavior. This basic paradigm has been used extensively in recent years to delineate the neural circuitry important for these two classes of behavior. From such studies, regions of the amygdala, dorsal striatum, and prefrontal cortex have been identified as critical neural substrates of these behavioral processes (Balleine et al., 2003; Coutureau and Killcross, 2003; Yin et al., 2004). A recent article in The Journal of

Received March 12, 2012; revised April 12, 2012; accepted April 13, 2012. Correspondence should be addressed to Jean-Marie N. Maddux, Department of Psychological and Brain Sciences, Johns Hopkins University, 3400 North Charles Street, Baltimore, MD 21218. E-mail:j.m.maddux@jhu.edu. DOI:10.1523/JNEUROSCI.1223-12.2012

Copyright $\odot 2012$ the authors $\quad 0270-6474 / 12 / 327769-02 \$ 15.00 / 0$
Neuroscience by Lingawi and Balleine (2012) builds upon this work and furthers our understanding of the neural circuits underlying habitual instrumental behavior.

Using the methodology outlined above, Lingawi and Balleine (2012) identified the anterior portion of the amygdala central nucleus $(\mathrm{aCeN})$ as a necessary brain region for the acquisition of habits. All rats were overtrained on a lever-press response and, thereafter, the outcome was devalued using a conditioned taste-aversion procedure involving injections of $\mathrm{LiCl}$. When tested in extinction, rats with bilateral sham lesions or lesions of the posterior part of the amygdala central nucleus showed no effect of devaluation; that is, these rats displayed a response pattern that did not reflect the current value of the outcome. Habitual responding in these animals, then, was unperturbed. By contrast, rats with bilateral lesions of the aCeN maintained sensitivity to devaluation even after instrumental overtraining, indicated by responding that was determined by the current value of the outcome. Thus, habitual responding in these animals was impaired. Moreover, Lingawi and Balleine (2012) showed that interaction of the aCeN with the dorsolateral striatum (DLS), a region previously identified as important for habitual behavior (Yin et al., 2004), is needed for habit learning. That is, rats with contralateral lesions of aCeN and DLS, but not ipsilateral lesion controls, remained sensitive to devaluation, indicating a lack of habitual responding.

Lingawi and Balleine's (2012) findings are unique in their identification of aCeN, and its interaction with DLS, as important for habit learning. Indeed, these results contribute to a growing body of literature dissociating the roles of the basolateral amygdala (BLA) and CeN (Holland and Gallagher, 1999; Corbit and Balleine, 2005) by extending the distinction to goal-directed versus habitual behavior. Furthermore, this study reports a functional dissociation along the anterior-posterior dimension within $\mathrm{CeN}$, a finding that suggests the need for precise hodological considerations when surveying this structure and its heterogeneous function. Notably, projections from $\mathrm{CeN}$ to the substantia nigra pars compacta $(\mathrm{SNc})$ arise primarily from anterior portions of medial $\mathrm{CeN}$ (Gonzales and Chesselet, 1990; Wallace et al., 1992), and signals transmitted down these efferents may code prediction error information (Lee et al., 2010). If and how these projections modulate putative reinforcement signals found in midbrain dopaminergic neurons (Schultz and Dickinson, 2000) remains to be determined for a variety of learning conditions, including those for habits. Importantly though, while Lingawi and Balleine (2012) claim that aCeN is involved in the learning of habits, future studies are needed to disambiguate its role in the acquisition of the habitual instrumental response from the performance of that very response. Posttraining lesion or reversible inactivation of aCeN may be useful in this regard.

Some recent anatomical findings examining innervation of the intercalated cells (ITCs) of the amygdala by the medial pre- 
frontal cortex (Pinard et al., 2012) have particular relevance for the interpretation of Lingawi and Balleine's (2012) work. Lingawi and Balleine (2012) raise the possibility that the interaction between goal-directed and habitual responding may rely on the interaction of BLA and $\mathrm{CeN}$, which, if true, could be mediated by the ITCs of the amygdala. As the authors point out, the ITCs themselves seem to be regulated by the infralimbic cortex (IL). Interestingly, when IL is temporarily inactivated by muscimol infusion, rats exhibit reinstatement of goaldirected responding following instrumental overtraining (Coutureau and Killcross, 2003). Perhaps then, IL input to the ITCs mediates the balance between goal-directed and habitual responding. Notably though, recent anatomical work reports that the projections from IL to the ITCs are, in fact, light to moderate (Pinard et al., 2012). In contrast, Pinard et al. (2012) found a very dense projection from IL to the far lateral region of the capsular subdivision of the central nucleus, a region they termed the "capsular infralimbic target zone" (CITZ). If IL indeed mediates an interaction of goaldirected and habitual mechanisms subserved by amygdala subdivisions, these findings suggest that it would be able to do so in the absence of strong projections to the ITCs. Alternatively, given the dense projection from IL to the CITZ, it is possible that IL exerts this hypothesized regulatory influence on CeN directly, bypassing the ITCs entirely. That is, because the capsular subdivision, itself the most anterior portion of $\mathrm{CeN}$, demonstrates considerable intraconnectivity as well as moderate innervation of the entire rostrocaudal extent of the medial CeN (mCeN) (Jolkkonen and Pitkänen, 1998), an IL-CITZ - mCeN-SNc serial circuit is credible. These relationships need not be considered mutually exclusive, and further work needs to be done to clarify how IL may influence the amygdala during transitions between goal-directed and habitual behavior. Importantly, an asymmetrical lesion approach, as used by Lingawi and Balleine (2012), would not be helpful in this regard, because IL projections to the amygdaloid complex are bilateral (McDonald et al., 1996; Pinard et al., 2012). Instead, projection-selective approaches (e.g., using optogenetics) are required for investigations of communication between the brain regions.

Finally, Lingawi and Balleine's (2012) findings suggest another potentially fruitful line of research. In humans and rodents, stress can induce a shift from goal-directed to habitual instrumental behavior (DiasFerreira et al., 2009; Schwabe and Wolf, 2009). Furthermore, administration of the $\beta$-adrenoceptor antagonist propranolol can prevent that shift, thereby implicating noradrenergic involvement in stress-induced habitual control of instrumental action (Schwabe et al., 2011). Just as Lingawi and Balleine's (2012) pretraining aCeN lesion resulted in rats that remained sensitive to outcome devaluation following extensive instrumental training, humans that received propranolol before a stress manipulation remained goal-directed in their behavior, as assessed by instrumental devaluation (Schwabe et al., 2011). Moreover, through reciprocal relations with the brainstem noradrenergic system, neurons in $\mathrm{CeN}$ that release corticotropin-releasing factor may drive the stress circuitry subserving motivational withdrawal from drugs of abuse (Koob, 2009). Through its influence on withdrawal, CeN may have a critical role in addiction, the progression of which is thought to reflect initial voluntary drug use, a type of goal-directed behavior that eventually transitions to habitual drug use, in which the addict experiences a loss of control (Everitt and Robbins, 2005; Everitt et al., 2008).

Now that Lingawi and Balleine (2012) have specifically identified aCeN as another brain region involved in habitual behavior, the interplay of stress peptides in this area may be particularly useful in furthering our understanding of not only the learning processes guiding instrumental conditioning, but also of neural and behavioral dysfunction as they relate to socially important issues, such as drug addiction. Acquisition of habit learning with drug rewards has been shown to be regulated by dopaminedependent spiraling projections through the midbrain that connect the ventral striatum with the dorsal striatum (Belin and Everitt, 2008; Everitt et al., 2008). In particular, projections from the nucleus accumbens core to the DLS through the substantia nigra have been shown to be critical in habitual responding on a second-order schedule of cocaine reinforcement (Belin and Everitt, 2008; Everitt et al., 2008). Future experiments should consider whether the aCeN and its connections with the DLS contribute to habit learning in a similar manner with drug rewards (e.g., cocaine) as it does with natural rewards.

\section{References}

Adams CD (1982) Variations in the sensitivity of instrumental responding to reinforcer devaluation. Q J Exp Psychol B 34:77-98.

Balleine BW, Killcross AS, Dickinson A (2003) The effect of lesions of the basolateral amygdala on instrumental conditioning. J Neurosci 23:666-675.

Belin D, Everitt BJ (2008) Cocaine seeking habits depend upon dopamine-dependent serial connectivity linking the ventral with the dorsal striatum. Neuron 57:432-441.

Corbit LH, Balleine BW (2005) Double dissociation of basolateral and central amygdala lesions on the general and outcome-specific forms of Pavlovian-instrumental transfer. J Neurosci 25:962-970.

Coutureau E, Killcross S (2003) Inactivation of the infralimbic prefrontal cortex reinstates goal-directed responding in overtrained rats. Behav Brain Res 146:167-174.

Dias-Ferreira E, Sousa JC, Melo I, Morgado P, Mesquita AR, Cerqueira JJ, Costa RM, Sousa N (2009) Chronic stress causes frontostriatal reorganization and affects decision-making. Science 325:621-625.

Everitt BJ, Robbins TW (2005) Neural systems of reinforcement for drug addiction: from actions to habits to compulsion. Nat Neurosci 8:1481-1489.

Everitt BJ, Belin D, Economidou D, Pelloux Y, Dalley JW, Robbins TW (2008) Neural mechanisms underlying the vulnerability to develop compulsive drug-seeking habits and addiction. Philos Trans R Soc Lond B Biol Sci 363:3125-3135.

Gonzales C, Chesselet MF (1990) Amygdalonigral pathway: an anterograde study in the rat with Phaseolus vulgaris leucoagglutinin (PHA-L). J Comp Neurol 297:182-200.

Holland PC, Gallagher M (1999) Amygdala circuitry in attentional and representational processes. Trends Cogn Sci 3:65-73.

Jolkkonen E, Pitkänen A (1998) Intrinsic connections of the rat amygdaloid complex: projections originating in the central nucleus. J Comp Neurol 395:53-72.

Koob GF (2009) Brain stress systems in the amygdala and addiction. Brain Res 1293:61-75.

Lee HJ, Gallagher M, Holland PC (2010) The central amygdala projection to the substantia nigra reflects prediction error information in appetitive conditioning. Learn Mem 17:531-538.

Lingawi NW, Balleine BW (2012) Amygdala central nucleus interacts with dorsolateral striatum to regulate the acquisition of habits. J Neurosci 32:1073-1081.

McDonald AJ, Mascagni F, Guo L (1996) Projections of the medial and lateral prefrontal cortices to the amygdala: a Phaseolus vulgaris leucoagglutinin study in the rat. Neuroscience 71:55-75.

Pinard CR, Mascagni F, McDonald AJ (2012) Medial prefrontal cortical innervation of the intercalated nuclear region of the amygdala. Neuroscience 205:112-124.

Schultz W, Dickinson A (2000) Neuronal coding of prediction errors. Ann Rev Neurosci 23:473-500.

Schwabe L, Wolf OT (2009) Stress prompts habit behavior in humans. J Neurosci 29:7191-7198.

Schwabe L, Höffken O, Tegenthoff M, Wolf OT (2011) Preventing the stress-induced shift from goal-directed to habit action with a beta-adrenergic antagonist. J Neurosci 31:17317-17325.

Wallace DM, Magnuson DJ, Gray TS (1992) Organization of amygdaloid projections to brainstem dopaminergic, noradrenergic, and adrenergic cell groups in the rat. Brain Res Bull 28:447-454.

Yin HH, Knowlton BJ, Balleine BW (2004) Lesions of dorsolateral striatum preserve outcome expectancy but disrupt habit formation in instrumental learning. Eur J Neurosci 19:181-189. 